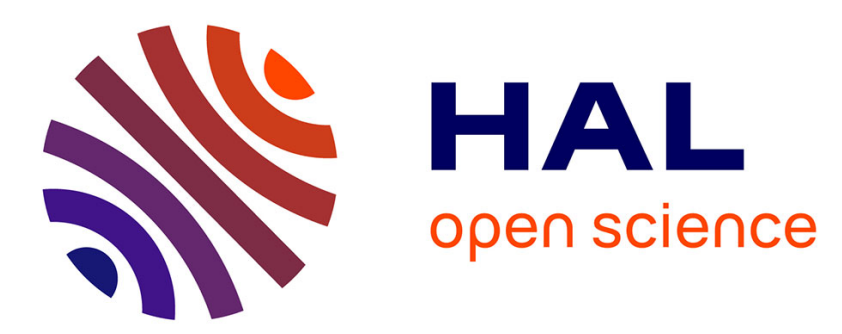

\title{
ANALYSE DU RAYONNEMENT ACOUSTIQUE D'UNE PLAQUE AVEC CONTROLE ACTIF DE L'AMPLITUDE DES PREMIERS MODES
}

A. Frances, Grégory Chatel, A. Roure

\section{- To cite this version:}

A. Frances, Grégory Chatel, A. Roure. ANALYSE DU RAYONNEMENT ACOUSTIQUE D'UNE PLAQUE AVEC CONTROLE ACTIF DE L'AMPLITUDE DES PREMIERS MODES. Journal de Physique Colloques, 1990, 51 (C2), pp.C2-205-C2-208. 10.1051/jphyscol:1990249 . jpa-00230670

\section{HAL Id: jpa-00230670 https://hal.science/jpa-00230670}

Submitted on 1 Jan 1990

HAL is a multi-disciplinary open access archive for the deposit and dissemination of scientific research documents, whether they are published or not. The documents may come from teaching and research institutions in France or abroad, or from public or private research centers.
L'archive ouverte pluridisciplinaire HAL, est destinée au dépôt et à la diffusion de documents scientifiques de niveau recherche, publiés ou non, émanant des établissements d'enseignement et de recherche français ou étrangers, des laboratoires publics ou privés. 
COLLOQUE DE PHYSIQUE

Colloque C2, supplément au $n^{\circ} 2$, Tome 51, Février 1990

ler Congrès Français d'Acoustique 1990

\title{
ANALYSE DU RAYONNEMENT ACOUSTIQUE D'UNE PLAQUE AVEC CONTROLE ACTIF DE
} L'AMPLITUDE DES PREMIERS MODES

\author{
A. Frances, G. CHATEL* et A. ROURE** \\ CERDAN (Centre $d$ 'Etudes et de Recherches pour la Discrétion Acoustique \\ des navires), DCN Toulon, F-83800 Toulon Naval, France \\ * SECAV (Société Etude et Contrôle en Acoustique et Vibration). \\ 6 Boulevard de Brazza, F-13008 Marsellie, France \\ * CNRS, Laboratoire de Mécanique et d'Acoustique, 31, Chemin Joseph \\ Aiguier, F-13009 Marseille, France
}

\begin{abstract}
Résumé - Le rayonnement d'une structure métallique complexe, excitée par un champ acoustique diffus est analysé expérimentalement en utilisant une approche modale. On montre la prédominance des premiers modes propres de la structure dans la puissance acoustique rayonnée. II est développé ensuite une méthode d'isolation active, utilisant un faible nombre d'actuateurs et permettant une atténuation importante de la puissance acoustique rayonnée. L'analyse du comportement mécano-acoustique de la paroi, contrôlèe par le système d'isolation active, montre d'une part, une amplification des modes résonants et d'autre part, une diminution importante de l'amplitude des modes rayonnants.
\end{abstract}

\begin{abstract}
The radiation efficiency of a stiffened steel plate is examined according to the modal analysis of the plate. The plate's acoustic radiation comes mainly from the amplitude of the structure's first modes. An insulation method is developed based on amplitude control of the first modes by applying active inputs. This insulation method is applied to the studied plate and the acoustic radiation reduction is examined according to the modal analysis of the plate.
\end{abstract}

\section{1 - INTRODUCTION}

L'isolation en basses fréquences d'une plaque mince soumise à un champ acoustique diffus est un problème difficile à résoudre par l'utilisation d'une méthode d'isolation passive. Pour une plaque rectangulaire, et pour des fréquences inférieures à la fréquence de coincidence WALLACE/1/ a montré la dominance des premiers modes impairs dans l'efficacité au rayonnement.

Une plaque métallique soumise à une excitation acoustique, répond simultanément sur un grand nombre de modes. De ce fait, il nous a paru nécessaire pour obtenír une meilleure compréhension du mécanisme du rayonnement acoustique d'une paroi raidie, de procéder :

- à l'analyse modale de la réponse de la paroi excitée en champ diffus;

- au classement de la puissance acoustique rayonnée par chacun des modes et par fréquence;

- à la détermination de l'atténuation du bruit rayonné par la paroi, par la suppression des premiers modes.

A partir de l'analyse effectuée, il nous a paru possible de développer une méthode d'isolation active basée sur le contrôle de l'amplitude des modes rayonnants. 


\section{2 - CONFIGURATIONS EXPERIMENTALES}

L'ensemble des mesures a été réalisé sur une paroi métallique raidie, montée verticalement dans la baie de la station d'essais du L.M.A. Cette paroi métallique d'épaisseur $3 \mathrm{~mm}$ et d'une surface de $10 \mathrm{~m}^{2}(2,46 \times 3,96 \mathrm{~m})$, séparait une salle réverbérante et une salle de réception.

Les vitesses normales de la plaque ont été mesurées en 442 points distribués suivant un maillage carré, du côté de la salle de réception. Les intensités rayonnées ont été mesurées en 104 points répartis sur la surface de mesurage à $50 \mathrm{~cm}$ en avant de la paroí.

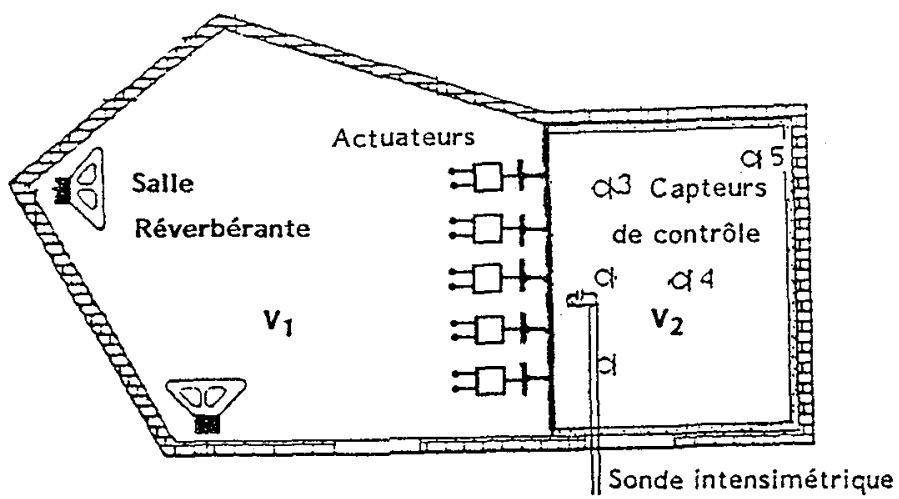

Fig.1 - Montage expérimental -

Système d'isolation active comprenant cinq actuateurs et cinq capteurs de contrôle.

\section{3 - ANALYSE DU RAYONNEMENT DE LA PAROI SOUMISE A UNE EXCITATION ACOUSTIQUE}

Le déplacement vibratoire de la plaque excitée par des sources acoustiques positionnées dans le volume $V 1$ ainsi que par une distribution de forces mécaniques $F$, normales à la plaque aux points $Q_{0}$, est solution de l'équation de propagation suivante :

$$
L W(Q)+m(Q) \frac{\partial^{2} W(Q)}{\partial t^{2}}=F S\left(Q-Q_{0}\right)+P_{1}(Q)-P_{2}(Q)
$$

avec :

L : Opérateur différentiel en rapport avec la rigidité de flexion de la plaque

m : masse surfacique de la plaque

$W(Q)$ : déplacement normal à la structure $S$

P1 - P2 : distribution des pressions sur la surface de la plaque

L'intégrale de distribution des pressions sur la surface de la plaque donne des facteurs de participation modale $a_{n, m}(t)$ complexes en fonction du temps. La déformée de la structure s'écrit comme suit :

$$
W(Q, t)=\sum_{n=1}^{\infty} \sum_{m=1}^{\infty}\left|a_{n, m}\right| e^{j\left(\omega t+\varphi_{n, m}\right)} \Psi_{n, m}(Q)
$$

$\varphi_{n, m}$ est la phase temporelle associée au mode $\Psi_{n, m}$

La puissance acoustique émise par la paroi ou par chacun de ses modes pris séparément, est ensuite déterminée par le calcul du flux d'énergie à travers une surface de mesurage fermée sur la paroi. 
- Résultats : Les déformées vibratoires de la structure, excitée par un champ diffus, sont dominées par des modes rẻsonants présentant une faible efficacité au rayonnement.

Le mode $(1,1)$ est dominant dans la puissance acoustique rayonnée, en basses fréquences et jusqu'à 60 Hertz. Pour les fréquences comprises entre 60 et $120 \mathrm{Hertz}$, la puissance acoustique provient essentiellement de la combinaison des trois premiers modes $(1,1),(1,2)$ et $(2,1)$.

La minimisation du rayonnement de la plaque est ensuite étudiée avec l'utilisation d'un faible nombre d'actuateurs convenablement positionnés pour agir sur les modes rayonnants.

4 - METHODE DE L'ISOLATION ACTIVE DE LA PAROI

II a été mis en oeuvre une méthode dite de synthèse synchrone, dans laquelle le champ primaire est constitué de fréquences pures stationnaires et les signaux de contre-vibration sont envoyés sur les actuateurs, en synchronisme avec le champ primaire, par l'intermédiaire d'un synthétiseur programmable. Les signaux envoyés sont de la forme : $\mathbf{U}(\mathrm{f})=\left[\mathbf{H}^{*} \mathbf{H}\right]^{-1} \cdot \mathbf{H}^{*} \cdot \mathbf{P}^{\mathbf{0}}(\mathrm{f})$

où $H(f)$ est la matrice de transfert entre les actuateurs et les capteurs de contrôle et $\mathrm{P}^{\circ}(f)$ le champ de pression acoustique transmis par la paroi en l'absence de système d'isolation active.

Les essais d'absorption active ont été effectués sur des sons purs entre 40 et 60 Hertz avec trois puis cinq actuateurs positionnés convenablement sur les raidisseurs. Les capteurs de contrôle servant à la minimisation étaient cinq microphones répartis devant la cloison et dans la salle de réception.

\section{5 - RESULTATS}

Le fonctionnement du dispositif d'isolation active, à la fréquence de $48 \mathrm{Hertz}$, se traduit par une diminution de 15 décibels du niveau d'intensité moyen mesuré en 104 points devant la paroi.

La figure 2 visualise, d'une part, la diminution de la puissance acoustique rayonnée par la paroi et d'autre part, l'augmentation des déformées vibratoires sur des modes complexes.

L'analyse modale des déformées de la paroi met en évidence, une diminution importante du premier mode de l'ordre de 8 décibels et une augmentation généralement supérieure de plus de 10 décibels des autres modes. Ces résultats ont été vérifiés pour d'autres fréquences comprises entre 40 et 60 Hertz.

\section{6 - CONCLUSIONS}

Cette étude montre la faisabilité d'une méthode de minimisation active du rayonnement acoustique d'une structure complexe et de grandes dimensions, soumise à une excitation par un champ acoustique diffus. La méthode utilise un faible nombre d'actuateurs et a permis d'obtenir une atténuation de plus de 10 décibels dans la bande de fréquence à l'intérieur de laquelle les premiers modes ont une part dominante dans le rayonnement.

\section{REFERENCES}

11/ WALLACE, C.E, "Radiation resistance of a rectangular panel"

J.A.S.A. VOL 53 (3), part 2, p 946-952, 1972

12/ MAIDANIK G., "Response of ribbed panels to reverberant acoustic fields"

J.A.S.A. VOL 34 (3), 809-826, 1962

13/ FAHY F.J., "SOUND INTENSITY" - ELSEVIER APPLIED SCIENCE

14/ CHATEL G., and FRANCES A., "Confinement effect of a sound source on the sound power radiated by a rectangular plate. Experimental approach using intensity measurement" Noise-con 87, p 557-562, 1987 


\section{DISPOSITIF DE CONTROLE ACTIF A L'ARRET}

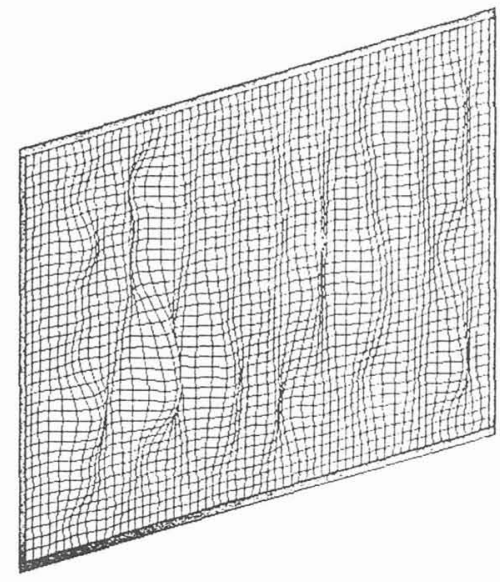

DEFORMEE INSTANTANEE

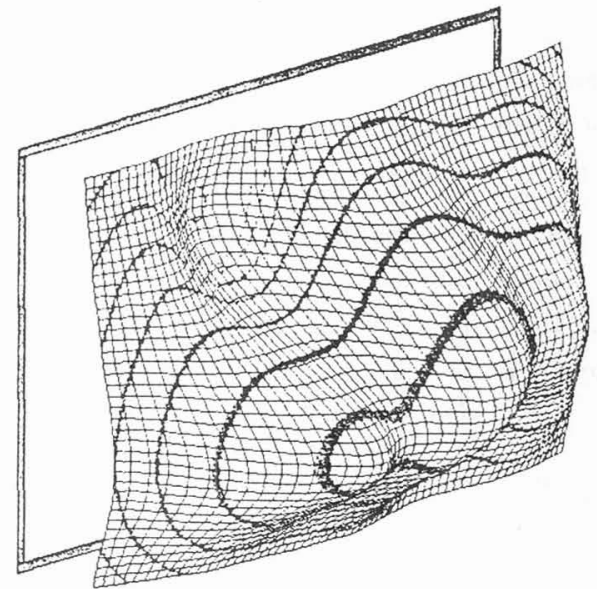

INTENSITE ACTIVE A 50 CM

DISPOSITIF DE CONTROLE ACTIF EN FONCTIONNEMENT

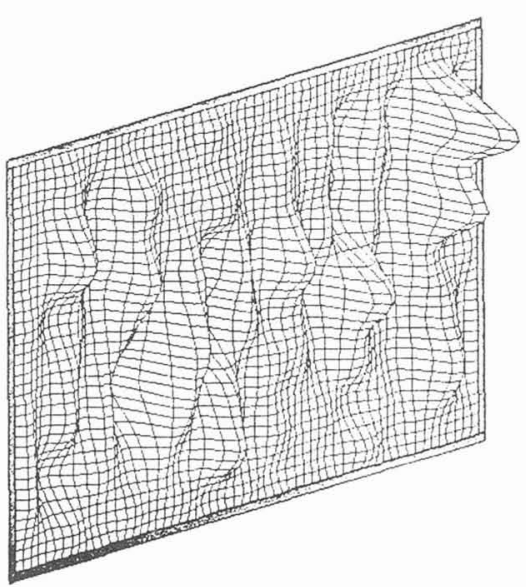

DEFORMEE INSTANTANEE

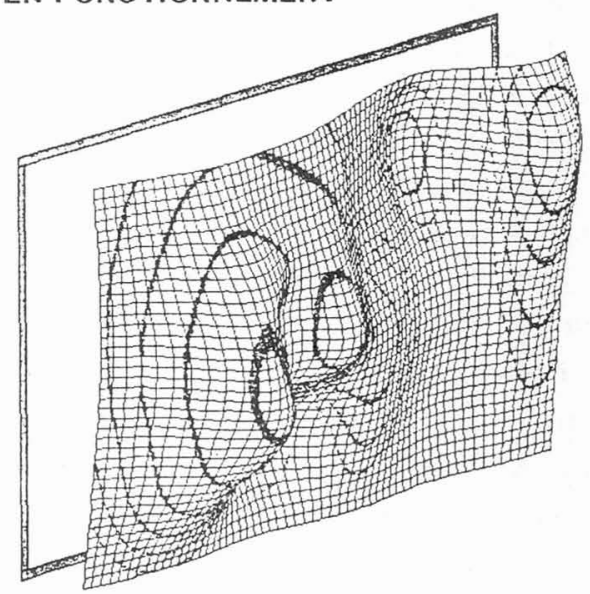

INTENSITE ACTIVE A 50 CM

FREOLENCE : $49 \mathrm{~Hz}$

Fig. 2 - Représentation de la déformée de la paroi et de la distribution de l'intensité acoustique rayonnée. La paroi est soumise à une excitation par un champ diffus à la fréquence de 48 Hertz. 